\title{
IMPROVED RESULTS ON THE MERTENS CONJECTURE
}

\author{
YANNICK SAOUTER AND HERMAN TE RIELE
}

\begin{abstract}
In this article, we study the Mertens conjecture. We revisit and improve the original constructive disproof of János Pintz. We obtain a new lower bound for the minimal counterexample and new numerical results for this conjecture.
\end{abstract}

\section{INTRODUCTION AND RESULTS}

The Mertens conjecture Mer97 states that

$$
|M(x)| / \sqrt{x}<1 \text { for } x>1,
$$

where $M(x)=\sum_{1 \leq n \leq x} \mu(n)$ and $\mu(n)$ is the Möbius function with values $\mu(1)=1$, $\mu(n)=(-1)^{k}$ if $n$ is the product of $k$ distinct primes, and $\mu(n)=0$ otherwise. The truth of the Mertens conjecture - but also the weaker assumption: $|M(x)| / \sqrt{x}<C$ for $x>1$ and some $C>1$-would imply the truth of the Riemann hypothesis. The Mertens conjecture was disproved by Odlyzko and te Riele in 1985 [OtR85] when they proved the existence of some $x$ for which $M(x) / \sqrt{x}>1.06$, and some other $x$ for which $M(x) / \sqrt{x}<-1.009$. The best recent result in this direction is due to Andreas Decker Dec10] who showed that limsup $\pm M(x) / \sqrt{x}>1.5$.

In 1987, Pintz Pin87] effectively disproved the Mertens conjecture by showing that $|M(x)| / \sqrt{x}>1$ for some $x \leq \exp \left(3.21 \times 10^{64}\right)$. In this paper we revisit and improve the original effective disproof of Pintz. A new lower bound of $\exp (1.004 \times$ $10^{33}$ ) for the minimal counterexample is established and new numerical results for the Mertens conjecture are obtained.

\section{The Results of Pintz}

For a more comprehensive exposition, we will adopt the notation of Pintz's paper [Pin87]. The sequence of complex numbers $\rho_{\nu}=\beta_{\nu}+i \gamma_{\nu}$ will denote the sorted list of non-trivial zeros of the Riemann function $\zeta$ belonging to the upper half complex plane $\left(0<\gamma_{1}<\gamma_{2}<\ldots\right)$. Values $\psi_{\nu}$ are then defined such that $\pi \psi_{\nu}=\operatorname{Arg}\left(\rho_{\nu} \zeta^{\prime}\left(\rho_{\nu}\right)\right)$. The sum $K_{2}(v, T, k)$, where $v, T$ and $k$ are positive, is defined by

$$
K_{2}(v, T, k):=2 \sum_{0<\gamma_{\nu}<T} e^{-k \gamma_{\nu}^{2}} \frac{\cos \left(\gamma_{\nu} v-\pi \psi_{\nu}\right)}{\left|\rho_{\nu} \zeta^{\prime}\left(\rho_{\nu}\right)\right|} .
$$

Note that this definition is meaningful if and only if the zeros of the Riemann function $\zeta$ are all simple, at least up to height $T$. The main theorem of [Pin87] is the following.

Received by the editor December 14, 2011 and, in revised form, April 26, 2012 and May 9, 2012.

2010 Mathematics Subject Classification. Primary 11-04, 11A15, 11M26, 11Y11, 11 Y35.

(C)2013 American Mathematical Society 
Theorem A. If there exists a value $v \in\left[e^{7}, e^{5 \times 10^{4}}\right]$ such that

$$
\left|K_{2}\left(v, 1.4 \times 10^{4}, 1.5 \times 10^{-6}\right)\right|>1+e^{-40},
$$

then there exists $x$ with $1 \leq x \leq e^{v+\sqrt{v}}$ and $|M(x)|>\sqrt{x}$.

The effective disproof of the Mertens conjecture was finally obtained by computing the left-hand side of (2.2) for a value $v \simeq 3.2097 \times 10^{64}$. This value has been determined by Odlyzko and te Riele OtR85] and, numerically we have $K_{2}\left(v, 1.4 \times 10^{4}, 1.5 \times 10^{-6}\right) \simeq-1.00223$. Theorem 2 of [Pin87] is then:

\section{Theorem B.}

$$
\max _{x \leq X} \frac{|M(x)|}{\sqrt{x}}>1 \text { if } X=\exp \left(3.21 \times 10^{64}\right) .
$$

Kotnik and te Riele KtR06 extended these computations and were able to find lower values for $X$ in Theorem $\mathrm{B}$. During their search, they also found near miss values for $X$, i.e., values which were very likely to be smaller counterexamples but for which (2.2) does not hold. The main reason for this latter fact is that the value $k=1.5 \times 10^{-6}$ was too large. Indeed, in (2.1), the terms of the summation become negligible when the index $\gamma_{\nu}$ is large. Thus these terms do not contribute sufficiently to the final sum so that these near miss values for $X$ cannot be confirmed as counterexamples.

This value for $k$ was used in the original disproof and is a consequence of the limited computational power available in 1987. Indeed, as we will see below, if we wish to confirm counterexamples, lowering $k$ requires increasing $T$. A larger number of summation terms is then involved. Moreover, because of the large values for eventual candidates $v$, these terms have to be computed with great accuracy. This fact, in turn, requires great accuracy for the zeros $\rho_{\nu}$ of the Riemann function $\zeta$, and as we will see below, the computational load of this task increases rapidly with the size of $\gamma_{\nu}$.

Another point with (2.2) is that it has been derived using a computation of zeros of the Riemann function $\zeta$ performed by Rosser, Yohe and Schoenfeld [RYS69] in 1969. Pintz, in his derivation, used the minimal distance between consecutive zeros obtained in that paper, without information on the exact location of close zeros. As a consequence, one integral in the original work had to be bounded as if the worst case was occurring everywhere in the path of integration. As we will see below, with the knowledge of the explicit values of the zeros of the Riemann function $\zeta$, this integral can be bounded more efficiently and the computational cost can be reduced.

\section{Outline of the Proof}

Pintz's results are obtained by contradiction. In the first case, the Riemann hypothesis is supposed to be false. The following theorem can then be proven:

Theorem C. Let $\rho_{0}=\beta_{0}+i \gamma_{0}$ be a non-trivial zero of the Riemann function $\zeta$ with $\beta_{0}>1 / 2$ and $\gamma_{0}>0$. Then

$$
D(Y)=\frac{1}{Y} \int_{0}^{Y}|M(x)| d x>\frac{Y^{\beta_{0}}}{\gamma_{0}^{5}} \text { for } Y>\gamma_{0}^{5} .
$$

It is then straightforward to prove the following corollary: 
Corollary D. Let $Y>0$ and $\varepsilon>0$. Suppose that there exists a non-trivial zero $\rho_{0}$ of the Riemann function $\zeta$ with $\beta_{0}>\frac{1}{2}+\frac{5 \log \left|\gamma_{0}\right|+\varepsilon}{\log Y}$. Then $D(Y)>e^{\varepsilon} \sqrt{Y}$.

In Pin87, this latter result is stated in the case $\varepsilon=0$. This new version will permit us to obtain sharper estimates for the size of the Mertens function. In the following this result will be used with $\varepsilon=1$, which will be sufficient for our purpose.

At this point, two mean values of the Mertens function are introduced for positive $u$ and $k$ :

$$
\begin{gathered}
M_{1}(u)=\frac{e^{-k / 4-u / 2}}{2 \sqrt{\pi k}} \int_{u-2 \sqrt{k u}}^{u+2 \sqrt{k u}} \frac{M(x)}{x} \exp \left(-\frac{(u-\log x)^{2}}{4 k}\right) d x \\
M_{2}(u)=\frac{e^{-k / 4-u / 2}}{2 \sqrt{\pi k}} \int_{1}^{+\infty} \frac{M(x)}{x} \exp \left(-\frac{(u-\log x)^{2}}{4 k}\right) d x
\end{gathered}
$$

The following lemmas are proven:

Lemma E. If $\mid M\left(x \mid \leq A \sqrt{x}\right.$ for $|\log x-u| \leq 2 \sqrt{k u}$, then $\left|M_{1}(u)\right|<A$.

Lemma F. $\left|M_{2}(u)-M_{1}(u)\right|<2 e^{-u / 4}$ if $k<1 / 4$ and $u \geq 16$.

Lemma G. $M_{2}(u)=\frac{e^{-k / 4-u / 2}}{2 \pi i} \int_{2-i \infty}^{2+i \infty} \frac{e^{k s^{2}+u s}}{s \zeta(s)} d s$.

Lemma E provides by contradiction a criterion on $M_{1}(u)$ to prove the existence of values for $M(x) / \sqrt{x}$ larger than $A$. Lemma F proves that, under some conditions, values of $M_{1}(u)$ and $M_{2}(u)$ are very close. We are then left with the study of $M_{2}(u)$ and Lemma G gives an alternate representation of $M_{2}(u)$. By the use of the residue theorem, it is possible to modify the original integration path of Lemma $G$ and we have:

Lemma H. Let $u>2$ and $k>0, T_{1}>T_{0}>0$. Suppose that the Riemann hypothesis holds up to at least height $T_{0}$. Then we have:

$$
M_{2}(u)=\frac{1}{2 \pi i} \int_{(L)} \frac{e^{k\left(s^{2}-1 / 4\right)+u(s-1 / 2)}}{s \zeta(s)} d s+K_{2}\left(u+k, T_{0}, k\right)
$$

where the integration path $L$ is formed from $L_{1}, L_{2}, L_{3}, L_{4}, L_{5}, L_{6}$ and their reflected image with the real axis, where we define:

$$
\begin{aligned}
& L_{1}=\left\{s=\frac{1}{4}+i t \mid 0 \leq t \leq T_{0}\right\} \\
& L_{2}=\left\{s=\sigma+i T_{0} \mid \frac{1}{4} \leq \sigma \leq \frac{1}{2}+\frac{1}{u}\right\} \\
& L_{3}=\left\{s=\frac{1}{2}+\frac{1}{u}+i t \mid T_{0} \leq t \leq T_{1}\right\} \\
& L_{4}=\left\{s=\sigma+i T_{1} \mid \frac{1}{2}+\frac{1}{u} \leq \sigma \leq \frac{1}{2}+\frac{5 \log T_{1}+3}{u}\right\} \\
& L_{5}=\left\{s=\sigma+i t \mid t \geq T_{1}, \sigma \leq 2, \sigma=\frac{1}{2}+\frac{5 \log t+3}{u}\right\} \\
& L_{6}=\left\{s=2+i t \mid t \geq \exp \left(\frac{3}{10} u-\frac{3}{5}\right)\right\}
\end{aligned}
$$


The transformation of the integration path in Lemma $\mathrm{H}$ is almost the same as that of Lemma D in the original paper. There are, however, two differences. First, in paths $L_{4}$ and $L_{5}$, the numerators of the rightmost fractions are $5 \log T_{1}+2$ and $5 \log t+2$. In our paper, the value 2 is replaced by 3 since we are going to use Corollary $\mathrm{D}$ with $\varepsilon=1$. The second difference with Pintz's work is that we introduced the path $L_{6}$ which does not exist in the original paper. This point seems to be a minor error there. In fact, in Pin87, the path $L_{5}$ is unbounded in $\sigma$ while the author uses bounds on $\zeta(s)$ proven only for $0 \leq \sigma \leq 2$. Indeed, the following lemmas are used:

Lemma I. Let $s=\sigma+i$ with $0 \leq \sigma \leq 2$ and $t \geq 4$. If $\zeta(s) \neq 0$, then we have $\log \zeta(s)=\sum_{|\gamma-t| \leq c_{0}} \log (s-\rho)+\theta(11 \log t+14)$, where $\rho=\beta+i \gamma$ describes the set of non-trivial zeros of function $\zeta, c_{0}=\tan (1)$ and $\theta$ is a complex number depending on $s$ with $|\theta| \leq 1$.

Lemma J. Let $s=\sigma+i t$ with $0 \leq \sigma \leq 2$ and $t \geq 4$, and $v>0$. If $|s-\rho| \geq 1 / v$ for all zeros $\rho$ of function $\zeta$, then we have:

$$
\log |1 / \zeta(s)| \leq c_{0} \log t \log v+11 \log t+14
$$

The case where $|t| \leq 4$ is treated by the following lemma:

Lemma K. $|\zeta(1 / 4+i t)| \geq e^{-150}$ for $|t| \leq 4$.

\section{Generic Bounds FOR Integrals}

Lemmas 【and J can be used to obtain upper bounds on $|1 / \zeta(s)|$ provided that we have some information about the distribution of non-trivial zeros of $\zeta$. In turn, we can obtain upper bounds for the integral part of the right-hand side of (3.4). We then expect to show that the contribution of the integral term is negligible and thus to reduce the study of $M_{2}(u)$ to that of $K_{2}\left(u+k, T_{0}, k\right)$.

At this point, we make two additional assumptions on the zeros of the Riemann function $\zeta$. First, we suppose that the Riemann hypothesis holds in fact up to at least height $T_{1}$. Second, we suppose that for any zero $\rho_{\nu}$, we have $\beta_{\nu} \leq \frac{1}{2}+$ $\frac{5 \log \left|\gamma_{\nu}\right|+1}{\log u}$.

If this latter hypothesis is false, then Corollary $\mathrm{D}$ implies that $D(u)>e \sqrt{u}$. As a consequence, there exists at least one value with $1<x<u$ and $|M(x)| / \sqrt{x}>e$. If this hypothesis is true, it can be used to provide upper bounds on integrals. Let us denote $J_{i}=\int_{\left(L_{i}\right)} \frac{e^{k\left(s^{2}-1 / 4\right)+u(s-1 / 2)}}{s \zeta(s)} d s$ for $i \in[1 \ldots 6]$. Then we have:

$$
\left|M_{2}(u)-K_{2}\left(u+k, T_{0}, k\right)\right| \leq \frac{\left|J_{1}\right|+\left|J_{2}\right|+\left|J_{3}\right|+\left|J_{4}\right|+\left|J_{5}\right|+\left|J_{6}\right|}{\pi} .
$$


For integral $J_{1}$, we have $s=1 / 4+i t$ with $0 \leq t \leq T_{0}$, so that $1 /|s| \leq 4$. Moreover, Lemma J holds for $v=4$. So we have:

$$
\begin{aligned}
\left|J_{1}\right| \leq & 4 \int_{4}^{T_{0}} \exp \left(-\frac{3 k}{16}-k t^{2}-\frac{u}{4}+c_{0} \log t \log 4+11 \log t+14\right) d t \\
& +4 \int_{0}^{4} \exp \left(-\frac{3 k}{16}-k t^{2}-\frac{u}{4}+150\right) d t \\
\leq & 4 \exp \left(-\frac{u}{4}\right)\left(\int_{4}^{T_{0}} \exp \left(-k t^{2}+c_{0} \log T_{0} \log 4+11 \log T_{0}+14\right) d t\right. \\
& \left.\quad+\int_{0}^{4} \exp \left(-k t^{2}+150\right) d t\right) \\
\leq & 4 \exp \left(-\frac{u}{4}\right)\left(T_{0} \exp \left(14 \log T_{0}+14\right)+4 \exp (150)\right) \\
\leq & T_{0}^{15} \exp \left(-\frac{u}{4}+153\right) .
\end{aligned}
$$

For the integral $J_{2}$, we have $s=\sigma+i T_{0}$ with $1 / 4 \leq \sigma \leq 1 / 2+1 / u$, so that $1 /|s| \leq 1 / T_{0} \leq 1$. Up to height $T_{1}$, non-trivial zeros for $\zeta$ lie on the critical line. We put $\delta=\min \left(T_{0}-\gamma_{\nu_{0}}, \gamma_{\nu_{0}+1}-T_{0}\right)$, where $\nu_{0}$ is such that $\gamma_{\nu_{0}}<T_{0}<\gamma_{\nu_{0}+1}$. Then Lemma Dolds for $v=K$ where $K=1 / \delta$. If we suppose that $u>2$ and $0<k<1 / 4$, then we have:

$$
\begin{aligned}
\left|J_{2}\right| \leq & \int_{1 / 4}^{1 / 2+1 / u} \exp \left(k\left(\sigma^{2}-T_{0}^{2}-1 / 4\right)+u(\sigma-1 / 2)\right. \\
& \left.\quad+c_{0} \log T_{0} \log K+11 \log T_{0}+14\right) d \sigma \\
\leq & \exp \left(-k T_{0}^{2}-u / 2+c_{0} \log T_{0} \log K+11 \log T_{0}+14\right) \\
& \cdot \int_{1 / 4}^{1 / 2+1 / u} \exp \left(k \sigma^{2}+u \sigma\right) d \sigma \\
\leq & \exp \left(-k T_{0}^{2}-u / 2+c_{0} \log T_{0} \log K+11 \log T_{0}+14\right) \exp (u / 2+2) \\
\leq & \exp \left(-k T_{0}^{2}+c_{0} \log T_{0} \log K+11 \log T_{0}+16\right) .
\end{aligned}
$$

LemmaЏis not sufficient to bound efficiently the integral $J_{3}$. In the next section, we deal with this case with refined techniques.

For the integral $J_{4}$, we have $s=\sigma+i T_{1}$ with $1 / 2+1 / u \leq \sigma \leq 1 / 2+\left(5 \log T_{1}+3\right) / u$ and $1 /|s| \leq 1$. Lemma Jholds for this integral with $v=u$. For this case, we suppose that $\left(5 \log T_{1}+3\right) / u<1 / 2$ and $k<1$. Then we have: 


$$
\begin{aligned}
\left|J_{4}\right| \leq & \int_{1 / 2+1 / u}^{1 / 2+\left(5 \log T_{1}+3\right) / u} \exp \left(k\left(\sigma^{2}-T_{1}^{2}-1 / 4\right)+u(\sigma-1 / 2)\right. \\
& \left.+c_{0} \log T_{1} \log u+11 \log T_{1}+14\right) d \sigma \\
\leq & \exp \left(-k T_{1}^{2}-u / 2+c_{0} \log T_{1} \log u+11 \log T_{0}+14\right) \\
& \cdot \int_{1 / 2+1 / u}^{1 / 2+\left(5 \log T_{1}+3\right) / u} \exp \left(k \sigma^{2}+u \sigma\right) d \sigma \\
\leq & \exp \left(-k T_{1}^{2}-u / 2+c_{0} \log T_{1} \log u+11 \log T_{0}+14\right) \\
& \quad \cdot \frac{5 \log T_{1}+3}{u} \exp \left(u / 2+5 \log T_{1}+4\right) \\
\leq & \left(5 \log T_{1}+3\right) \exp \left(-k T_{1}^{2}+c_{0} \log T_{1} \log u+16 \log T_{1}+18\right) .
\end{aligned}
$$

For the integral $J_{5}$, we have $s=\frac{1}{2}+\frac{5 \log t+3}{u}+i t$ for $T_{1} \leq t \leq \exp \left(\frac{3}{10} u-\frac{3}{5}\right)$, so that $1 /|s| \leq 1$ and $|d s| \leq 2 d t$. Lemma J still holds for this integral with $v=u$, for example. Then we have:

$$
\begin{aligned}
\left|J_{5}\right| \leq \int_{T_{1}}^{\exp (3 u / 10-3 / 5)} \exp ( & k\left(-t^{2}-1 / 4\right)+5 \log t+3 \\
& \left.+c_{0} \log t \log u+11 \log t+14\right) d t \\
\leq & \exp (17) \int_{T_{1}}^{+\infty} \exp \left(-k t^{2}+16 \log t+c_{0} \log t \log u\right) d t
\end{aligned}
$$

Let us put $A=c_{0} \log u+16$. Suppose that $T_{1} \geq \sqrt{A / k}$ and $-\frac{k}{2} T_{1}^{2}+A \log T_{1} \leq 0$, then for $t \geq T_{1}$ the previous integrand is smaller than $\exp \left(-k t^{2} / 2\right)$. Then we have:

$$
\begin{aligned}
\left|J_{5}\right| & \leq \exp (17) \int_{T_{1}}^{+\infty} \exp \left(-k t^{2} / 2\right) d t \\
& \leq \exp (17) \frac{1}{\sqrt{k / 2}} \frac{\sqrt{\pi}}{2} \operatorname{erfc}\left(T_{1} \sqrt{k / 2}\right) \\
& \leq \frac{1}{k T_{1}} \exp \left(-\frac{k T_{1}^{2}}{2}+17\right)
\end{aligned}
$$

For the integral $J_{6}$, we have $s=2+i t$ for $t \geq \exp \left(\frac{3}{10} u-\frac{3}{5}\right)$. Lemma J holds for this integral with $v=1$, so that we have:

$$
\left|J_{6}\right| \leq \int_{\exp \left(\frac{3}{10} u-\frac{3}{5}\right)}^{+\infty} \exp \left(-k t^{2}+\frac{15 k}{4}+\frac{3 u}{2}+11 \log t+14\right) d t .
$$

Suppose that we have $\exp \left(\frac{3}{10} u-\frac{3}{5}\right) \geq \sqrt{11 / k}$ and $-\frac{k}{2} \exp \left(\frac{3}{5} u-\frac{6}{5}\right)+\frac{33}{10} u-\frac{33}{5} \leq 0$, then we have:

$$
\left|J_{6}\right| \leq \frac{1}{k \exp \left(\frac{3}{10} u-\frac{3}{5}\right)} \exp \left(-\frac{k \exp \left(\frac{3}{5} u-\frac{6}{5}\right)}{2}+\frac{3 u}{2}+15\right)
$$

\section{The CASE OF The PROBlematic INTEGRAL}

For practical candidates $u$, the previous upper bounds for integrals are infinitesimal or can be rendered negligible by appropriate choices for free variables. The case is a bit different for the integral $J_{3}$. Indeed, while Lemma J holds in this case 
for $v=u$, the derived bound is too large to enable final conclusions to be drawn. For this particular case, we need refined techniques.

For the moment, we suppose that the inequality $|\log (\zeta(s))| \leq \log (u)+11 \log t+M$ holds for $s$ in $L_{3}$ for an appropriate value of $M$. Then we have, for $|u| \geq 2$ and $k \leq 1 / 4$ :

$$
\left|J_{3}\right| \leq \int_{T_{0}}^{T_{1}} \exp \left(-k t^{2}+\log (u)+11 \log t+M+2\right) d t .
$$

If $T_{0} \geq \sqrt{11 / k}$ and $-\frac{k T_{0}^{2}}{2}+11 \log T_{0} \leq 0$, then we have:

$$
\left|J_{3}\right| \leq \frac{1}{k T_{0}} \exp \left(-\frac{k T_{0}^{2}}{2}+\log (u)+M+2\right) .
$$

The inequality and the corresponding value of $M$ required to derive this last inequality will be proven from Lemma \. First, for $s=\sigma+i t$, with $0 \leq \sigma \leq 2$ and $t \geq 4$, we have:

$$
\begin{aligned}
\log |\zeta(s)| & =\operatorname{Re}(\log (\zeta(s))) \\
& =\sum_{|\gamma-t| \leq c_{0}} \operatorname{Re}(\log (s-\rho))+\operatorname{Re}(\theta(11 \log t+14)) \\
& \geq \sum_{|\gamma-t| \leq c_{0}} \log |s-\rho|-11 \log t-14 .
\end{aligned}
$$

Now if $s$ belongs to $L_{3}$, we have $s=\frac{1}{2}+\frac{1}{u}+i t$ with $T_{0} \leq t \leq T_{1}$. Suppose that the Riemann hypothesis holds at least up to height $T_{1}+c_{0}$. Then any zero $\rho$ involved in the previous summation is such that $\rho=\frac{1}{2}+i \gamma$ with $T_{0}-c_{0} \leq \gamma \leq T_{1}+c_{0}$. Then we have $\log |s-\rho|=\log \left(\sqrt{1 / u^{2}+(t-\gamma)^{2}}\right)$. As a function of $t,|s-\rho|$ admits a global minimum equal to $-\log (u)$ for $t=\gamma$ and this function is stricly decreasing for $t \leq \gamma$ and strictly increasing for $t \geq \gamma$. Moreover, if $t \neq \gamma$, we have $\log |s-\rho| \geq \log |t-\gamma|$.

We define now $\theta_{\nu}=\frac{\gamma_{\nu}+\gamma_{\nu+1}}{2}$ and we suppose that $t \in\left[\theta_{\nu-1}, \theta_{\nu}\right]$. Then any zero $\rho=1 / 2+i \gamma$ of $\zeta$, such that $|\gamma-t| \leq c_{0}$ is such that $\theta_{\nu-1}-c_{0} \leq \gamma \leq \theta_{\nu}+c_{0}$.

First, if $\rho=1 / 2+i \gamma_{\nu}$, we have $\log |s-\rho| \geq-\log (u)$. Suppose now that $\rho=1 / 2+i \gamma_{\nu^{\prime}}$ with $\theta_{\nu-1}-c_{0} \leq \gamma_{\nu^{\prime}} \leq \theta_{\nu}+c_{0}$ and $\nu \neq \nu^{\prime}$. Then we have $\log |s-\rho|>\log \left|t-\gamma_{\nu^{\prime}}\right|$. If $\gamma_{\nu^{\prime}}<\gamma_{\nu}$, this gives $\log |s-\rho|>\log \left|\theta_{\nu-1}-\gamma_{\nu^{\prime}}\right|$, while if $\gamma_{\nu^{\prime}}>\gamma_{\nu}$, we have $\log |s-\rho|>\log \left|\theta_{\nu}-\gamma_{\nu^{\prime}}\right|$.

For a given $\rho=1 / 2+i \gamma_{\nu^{\prime}}$, the two previous lower bounds are effective for the summation of (5.3), for any $s=\frac{1}{2}+\frac{1}{u}+i t$ with $t \in\left[\theta_{\nu-1}, \theta_{\nu}\right]$ only if $\gamma_{\nu^{\prime}}-c_{0} \leq \theta_{\nu-1}$ and $\theta_{\nu} \leq \gamma_{\nu^{\prime}}+c_{0}$.

If one of these two conditions is not met, it means that the eventual lower bound is not valid for the whole interval. In this case, the corresponding $\log \left|\theta-\gamma_{\nu^{\prime}}\right|$ value is kept in the summation if it is negative. If it turns out to be positive, this value is discarded from the summation. Since we are looking for a global lower bound, this strategy gives a correct result for the entire interval.

Thus we have performed numerical computations in order to determine lower bounds of the expression $\sum_{|\gamma-t| \leq c_{0}} \log |s-\rho|$ of (5.3). The first 22 million intervals $\left[\theta_{\nu-1}, \theta_{\nu}\right]$ have been considered. The results are summarized in Table 1, In this table, column $N$ records successive extremal values for valid lower bounds of the summation. Thus the last value $N=-9.05$ is valid for the whole interval of computation, i.e., for $4 \leq t \leq 10379424.827854489$. The column $M$ then gives 
TABLE 1. Constants for lower bounds for $\log \left(\zeta\left(\frac{1}{2}+\frac{1}{u}+i t\right)\right)$ with $4 \leq t \leq 10379424.827854489$

\begin{tabular}{|c|c|c|}
\hline$\left[\theta_{\nu-1}, \theta_{\nu}\right]$ & $N$ & $M$ \\
\hline$[197268.960264916,197268.976758059]$ & -6.33 & 20.33 \\
\hline$[218899.718347201,218900.221843291]$ & -6.43 & 20.43 \\
\hline$[234016.329120325,234016.894982296]$ & -6.66 & 20.66 \\
\hline$[273192.971337030,273193.663137712]$ & -7.21 & 21.21 \\
\hline$[511464.060978107,511464.895650786]$ & -7.24 & 21.24 \\
\hline$[663317.570720223,663318.508310486]$ & -7.67 & 21.67 \\
\hline$[1961773.274790168,1961773.993356110]$ & -7.98 & 21.98 \\
\hline$[2159847.653552496,2159847.660178352]$ & -8.01 & 22.01 \\
\hline$[2218149.038583455,2218149.333203491]$ & -8.21 & 22.21 \\
\hline$[2650983.947015138,2650984.610665275]$ & -8.77 & 22.77 \\
\hline$[4316831.857820662,4316832.467101349]$ & -9.05 & 23.05 \\
\hline
\end{tabular}

the value to be used in (5.2). Values of Table 1 can then be used provided that $T_{1} \leq 10379424.827854489$.

Our computations enabled us to prove that $\log |1 / \zeta(s)| \leq \log (u)+11 \log t+23.05$ for $s=\frac{1}{2}+\frac{1}{u}+i t$ and $4 \leq t \leq 10^{7}$. As a comparison, the corresponding inequality derived by Pintz is found in [Pin87, Lemma 6] and asserts that $\log |1 / \zeta(s)| \leq$ $\log (u)+23.01 \log t+14$ for $4 \leq t \leq 1.1 \times 10^{6}$.

\section{Finding NEW COUntereXamples}

Eventual counterexamples to the Mertens conjecture can be found with the $L^{3}$ algorithm [LLL82 applied to an $(n+2) \times(n+2)$ matrix, the details of which are given in OtR85, (3.3)] and in [KtR06, (7)]. This results in an integer value $z$ with the property that many values of $\left(\gamma_{j} z / 1024-\pi \psi_{j}\right) \bmod 2 \pi$ for $1 \leq j \leq n$ in (2.1) are close to zero or to $2 \pi$, so that the corresponding $\cos \left(\gamma_{j} z / 1024-\pi \psi_{j}\right)$-values are close to 1 and the corresponding value of $K_{2}$ is relatively large. This matrix also depends on an integer parameter $\nu$ and in [KtR06] we have run the $L^{3}$ algorithm for all combinations $(\nu, n)$ in the range $\nu=8,9, \ldots, 400, n=[\nu / 4],[\nu / 4]+1, \ldots,[\nu / 2]$. For $\nu=133, n=57$ we found the 38D-integer

$$
z=36351141692560024009263302748479207105
$$

with $K_{2}^{*}(z)=K_{2}\left(z / 1024,1.4 \times 10^{4}, 1.5 \times 10^{-6}\right)=0.98856$ giving a near counterexample. This result was one of the motivations for the work in the present paper. In the meantime, we found a second, smaller, candidate counterexample, namely, for $\nu=130, n=45$, the 37D-integer

$$
z=1027817347878965798938101006330785076
$$

with $K_{2}^{*}(z)=-0.97553$.

Table 2 lists values of $z, \nu$, and $n$ which we have found for $|z|<10^{38}$ and $\left|K_{2}^{*}(z)\right|>0.95$. 
TABle 2. Possible counterexamples to the Mertens conjecture.

\begin{tabular}{|r|r|c|c|}
\hline$z$ & $\nu$ & $n$ & $K_{2}^{*}(z)$ \\
\hline 39755502637715984481041653607 & 101 & 40 & 0.95691 \\
-722697815468429397591634147185898 & 118 & 50 & 0.95819 \\
-11151302703816183589567459995538280 & 119 & 48 & -0.96787 \\
-37511058296151748679343309083664217 & 123 & 54 & 0.95999 \\
216072680367037700856984351791104824 & 125 & 48 & 0.95237 \\
1027817347878965798938101006330785076 & 130 & 45 & -0.97553 \\
1506726240378465647914443523724114901 & 128 & 44 & 0.95720 \\
2652469446976537245132684869611582627 & 127 & 43 & 0.97611 \\
3022614796664156290692018525628578321 & 130 & 52 & 0.97516 \\
3034722057812754549802547457921844038 & 133 & 63 & -0.96040 \\
-4347188671656452713195371881864617970 & 129 & 51 & 0.96901 \\
5258842403193137645778459939819416585 & 129 & 54 & -0.95532 \\
5769991780238851868089497487315551326 & 131 & 53 & -0.98410 \\
12531000768665507361151598018094973853 & 129 & 45 & 0.95729 \\
36202340664058586126784655122561392001 & 133 & 59 & 0.95589 \\
36351141692560024009263302748479207105 & 133 & 57 & 0.98856 \\
48783853562192888630207805798685191285 & 131 & 49 & 0.95612 \\
69217225631036739433556897835087389701 & 135 & 54 & 0.96118 \\
\hline
\end{tabular}

\section{Choosing constants}

From the preceding section, we have a possible counterexample $u=z / 1024 \approx$ $1.00372 \times 10^{33}$. The final conclusion will be drawn from the computation of $K_{2}(u+$ $\left.k, T_{0}, k\right)$ with appropriate values for $k$ and $T_{0}$. This computation will be validated provided that the upper bounds of the integrals $J_{1}, \ldots, J_{6}$ are small enough.

In our computations, we set $k=3 \times 10^{-9}$. For the 37D-integer $z$ of (6.1), we have $K_{2}\left(u+k, 1.4 \times 10^{4}, k\right)=-1.00738$. It was verified on the first zeros of $\zeta$, that larger values for $k$ would probably give values for $K_{2}\left(u+k, T_{0}, k\right)$ less than 1 in absolute value. From (4.8), we can easily prove that $\left|J_{6}\right| \leq \exp \left(-\exp \left(10^{30}\right)\right)$ and thus $J_{6}$ is infinitesimal.

From what precedes, we have seen that 10379424.827854489 is the maximal admissible value for $T_{1}$. Then we set $T_{1}=10^{7}$. Conditions are then satisfied for $J_{5}$ and (4.6) gives $\left|J_{5}\right| \leq \exp (-145000)$. Conditions are also satisfied for $J_{4}$ and, from (4.4), we obtain $\left|J_{4}\right| \leq \exp (-297000)$. We have also, necessarily, $T_{0} \leq T_{1}$, so that (4.2) can be used to prove $\left|J_{1}\right| \leq \exp \left(-10^{30}\right)$.

The previous integrals have extremely small values and thus do not influence the final computation. This is not the case for $J_{2}$ and $J_{3}$. Moreover, since their upper bounds involve $T_{0}$, in order to save computational cost it is better to choose a value for $T_{0}$ just large enough to validate the final computation. For $T_{0}=300000$, (5.3) gives $\left|J_{3}\right| \leq 10^{-11}$. The two zeros of $\zeta$ around height $T_{0}$ have heights 299999.720467800 and 300000.614026284. Thus we have $K \simeq 3.57$. Then (4.3) gives $\left|J_{2}\right| \leq 10^{-90}$.

As a consequence, we see that our choices $k=3 \times 10^{-9}, T_{0}=300000$ should be sufficient for the counterexample we wish to confirm. 


\section{Computing the summation}

From (2.1), we see that the exact computation of $K_{2}\left(v, T_{0}, k\right)$ for $v=u+k$ requires the computation of terms of the form $\cos \left(\gamma_{\nu} v-\pi \psi_{\nu}\right)$. Because of the large size of candidate $v$, this implies that the values $\gamma_{\nu}$ have to be determined with great accuracy. For the values $\psi_{\nu}$, the required accuracy is smaller because $-1<\psi_{\nu} \leq 1$. Then we need to compute the values $\gamma_{\nu}$ up to $T_{0}$ with great accuracy and the values $\zeta^{\prime}\left(1 / 2+\gamma_{\nu}\right)$ with less accuracy, at least in what concerns the numerator.

For the denominator, the values $\zeta^{\prime}\left(\rho_{\nu}\right)$ are again required. In theory, nothing prevents these values from being arbitrarily close to 0 . Thus, the absolute accuracy required for these values is a priori not known. However, in practice, these values are in fact quite far from 0 , so a reduced accuracy is sufficient.

The classical way to perform these kinds of computations is to use the EulerMaclaurin summation formula. For $k \leq 0$, we respectively denote $B_{k}$ and $B_{k}(x)$ the $k$-th Bernoulli number and the $k$-th Bernoulli polynomial. We also introduce the Pochhammer symbol $(s)_{m}=\Gamma(s+m) / \Gamma(m)$ and $(s)_{0}=1$ for $m$ an integer and $s$ a complex number. For any $s$ such that $\operatorname{Re}(s)>1-2 m$, for $m$ an integer with $m \geq 2$, and any integer $n \geq 1$, we then have, from [Cho95],

$$
\zeta(s)=\sum_{j=1}^{n} \frac{1}{j^{s}}+\frac{n^{1-s}}{s-1}-\frac{1}{2} n^{-s}+\sum_{k=1}^{m-1}(s)_{2 k-1} \frac{B_{2 k}}{(2 k) !} n^{-s-2 k+1}+R_{m}(s, n)
$$

where

$$
R_{m}(s, n)=(s)_{2 m} \int_{n}^{+\infty} \frac{B_{2 m}-B_{2 m}(x-[x])}{(2 m) !} x^{-s-2 m} d x
$$

and

$$
\begin{aligned}
\zeta^{\prime}(s) & =-\sum_{j=1}^{n} \frac{\log j}{j^{s}}-\frac{n^{1-s}}{(s-1)^{2}}-\frac{n^{1-s}}{s-1}\left(1-\frac{s-1}{2 n}\right) \log n \\
& -\sum_{k=1}^{m-1}(s+1)_{2 k-2} \frac{B_{2 k}}{(2 k) !} n^{-s-2 k+1}\left\{s \log n-1-\sum_{i=1}^{2 k-2} \frac{s}{s+i}\right\}+R_{m}^{\prime}(s, n)
\end{aligned}
$$

where

$$
\begin{aligned}
R_{m}^{\prime}(s, n)=-(s+1)_{2 m-1} \int_{n}^{+\infty} & \frac{B_{2 m}-B_{2 m}(x-[x])}{(2 m) !} \\
& \cdot\left\{s \log x-1-\sum_{i=1}^{2 k-1} \frac{s}{s+i}\right\} x^{-s-2 m} d x .
\end{aligned}
$$

These equations will be used for values on the critical line, i.e., for $s=\frac{1}{2}+i t, t>0$. Using this fact and properties of Bernoulli polynomials, with some computations it is possible to prove that in this case, we have

$$
\left|R_{m}(s, n)\right| \leq \frac{\pi^{2}}{3} \frac{(2 m-1+t)^{2 m-1} n^{-2 m+1 / 2}}{(2 \pi)^{2 m}}
$$


and

$$
\begin{aligned}
\left|R_{m}^{\prime}(s, n)\right| \leq & \frac{8(t+2 m)^{2 m-1}}{(2 \pi)^{2 m}(2 m-1 / 2) n^{2 m-1 / 2}} \\
& .\left((t+1)\left(\log n-\frac{1}{(2 m+1 / 2) n}\right)+2 m\right) .
\end{aligned}
$$

Given (8.5) and (8.6) and a desired accuracy $\eta>0$, it is then possible to choose values for $m$ and $n$ for practical computations. The strategy we used is the following. If $t \leq 5000$, then we fix $m=100$, else we put $m=\lfloor(t+1) / 50\rfloor$. Then $n$ is computed by dichotomy so that the right-hand side of (8.5) or (8.6) is less than $\eta$. This strategy gives a near optimal complexity in the sense that the number of terms to be considered in (8.1) and (8.3) grows nearly as $t /(2 \pi)$.

In [SD10], we already computed values of the first 22 million zeros of $\zeta$ to at least 9 correct decimal places of accuracy. First, we extended the accuracy of our zeros to at least 100 decimal places. For this purpose, we used the secant method. To reach this precision, 5 or 6 iterations of the secant method were generally sufficient. After this was done, we finally verified that at least 100 decimal places were indeed correct. To that end, we computed the values of $\zeta(s)$ with an offset of $5 \times 10^{-101}$ above and below the computed value to check the sign change. These values were then used to compute values for the derivative of $\zeta$ with (8.6). In this second computation, the final required accuracy was set to only 30 decimal places.

These computations had a high cost. Indeed, computing all the zeros $\rho$ and values $\zeta^{\prime}(\rho)$ up to height $T$ by Euler-Maclaurin summation has a complexity of $T^{2} \log (T)$, independently of the precision. Moreover, for the computation of the zeros, several summations are necessary. The cost is also linear with the required number of decimal places. These facts led us to realize that the direct computation of $K_{2}\left(u+k, T_{0}, k\right)$ with $T_{0}=300000$ would have been very lengthy.

Fortunately, it is possible to make shortcuts in the computations. Indeed, from (2.1), for $T_{0}^{\prime}<T_{0}$, we have:

$$
\left|K_{2}\left(v, T_{0}, k\right)-K_{2}\left(v, T_{0}^{\prime}, k\right)\right| \leq 2 \sum_{T_{0}^{\prime} \leq \gamma_{\nu}<T_{0}} \frac{e^{-k \gamma_{\nu}^{2}}}{\left|\rho_{\nu} \zeta^{\prime}\left(\rho_{\nu}\right)\right|} .
$$

The right-hand side of (8.7), that we denote by $K_{2}^{\prime}\left(T_{0}^{\prime}, T_{0}, k\right)$, is easier to compute since it does not require a large accuracy in the values of $\rho$ and $\zeta^{\prime}(\rho)$, provided that $T_{0}^{\prime}$ is large enough. Indeed, as said previously, the value of $\zeta^{\prime}(\rho)$ for $\rho$ being a zero of $\zeta$ is generally away from 0 . Thus the denominator of the fraction can be computed to several significant decimal places even if the precision of $\zeta^{\prime}(\rho)$ is not great, and the value of $\left|\rho \zeta^{\prime}(\rho)\right|$ can be expected to be large. On the contrary, the denominator for $\gamma>T_{0}^{\prime}$ is expected to be very small. As a consequence, the value of $K_{2}^{\prime}\left(T_{0}^{\prime}, T_{0}, k\right)$ is also expected to be small and it should be easily computed to, say, 8 or 9 digits of accuracy with limited computational cost.

We used again our database of zeros of $\zeta$ to compute values of $\left|\zeta^{\prime}(\rho)\right|$ up to $T=300000$. Since the precision in our database is certified to be at least 9 decimal digits after the decimal point, the precision of computed values is at least 3 decimal digits. The smallest value of $\left|\zeta^{\prime}(\rho)\right|$ was encountered for $\rho=\frac{1}{2}+i 78974.793348246$ and was evaluated to be equal to 0.121 when rounded to nearest. We can then affirm that $1 /\left|\zeta^{\prime}(\rho)\right| \leq 9$ for $\rho$ being a zero of $\zeta$ whose height is between 74000 and 300000. This upper bound was used in (8.7) in order to obtain a safe upper bound 
of the remainder sum. We finally obtain:

$$
\left|K_{2}^{\prime}(74000,300000, k)\right| \leq 5.69 \times 10^{-8} .
$$

On the other hand, using full precision computation of the zeros of $\zeta$ below $T_{0}^{\prime}=$ 74000 in (2.1), we obtain:

$$
K_{2}(v, 74000, k) \simeq-1.008867 .
$$

Thus we can finally assert the main result of this paper:

\section{Theorem L.}

$$
\max _{x \leq X} \frac{|M(x)|}{\sqrt{x}}>1.0088 \text { for } X=\exp \left(1.004 \times 10^{33}\right) .
$$

\section{Discussion}

In this paper, we have revisited the original explicit disproof of the Mertens conjecture given by Pintz Pin87]. As a result, we obtained a new lower bound for the first violation of the Mertens conjecture. This result has been obtained thanks to a better knowledge of zeros of the Riemann $\zeta$ function but also by the use of refined techniques to limit the required computational cost. Moreover, the bounds we used for the remainder terms of our computations are generic and can be used for further work on this topic.

A natural question arises: Is it still possible to improve on this result? In the authors' opinion, it is quite certain. As we have seen in Section 6, the new counterexample has been obtained by the use of the $L^{3}$ algorithm [LLL82. The principle of the search is to seek values of $v$ for which $\cos \left(\gamma_{\nu} v-\pi \psi_{\nu}\right)$ is close to 1 for as many as possible indices $\nu$ for which $\left|\rho_{\nu} \zeta^{\prime}\left(\rho_{\nu}\right)\right|^{-1}$ is large. This can be formulated as a problem of finding a shortest vector in a lattice. It is then solved with the use of the $L^{3}$ algorithm.

The counterexamples provided by the $L^{3}$ algorithm are extremely large. The exact growth of the Mertens function $M(x)$ is unknown but some conjectures have been made. For instance, Good and Churchhouse conjectured that

$$
\lim \sup |M(x)|(x \log \log x)^{-1 / 2}=\sqrt{12} / \pi .
$$

Independently, Kotnik and te Riele [KtR06] conjectured that

$$
\lim \sup |M(x)|(x \log \log \log x)^{-1 / 2}=1 / 2 .
$$

The first conjecture would place the first counterexample at $x \simeq 10^{10}$, which has been disproved. The second conjecture predicts the first counterexample to be at $x \simeq \exp \left(5 \times 10^{23}\right)$. Another conjecture was recently made. In Kac07, Kaczorowski gives some theoretical arguments that we could have in fact $|M(x)|=$ $\Omega\left(x^{1 / 2} \log \log \log x\right)$. By extrapolating from known values of $M(x)$, this latter conjecture would imply that the first violation occurs near $x \simeq 10^{703}$. These conjectures, although giving very different estimates, agree at least on the point that our counterexample is probably not the smallest one.

A further avenue of improvement would be to obtain stronger final results. Indeed, while we have obtained a new violation of Mertens conjecture, it should be emphasized that no numerical information is available for the smallest $x$ value such that $M(x)>\sqrt{x}$, or on the opposite side $M(x)<-\sqrt{x}$. It has yet to be noted that by virtue of the work of Odlyzko and te Riele [OtR85], such values are known 
to exist in both cases. Because of the dichotomic structure of the proof, we have information only about $|M(x)|$. For the same reason, although we can determine an upper bound, we cannot obtain a lower bound, while we would like to say that in fact $x \geq \exp (v-\sqrt{v})$. These two points might be treated if we were able to modify the path $L_{5}$ in such a way that it globally reaches the line $\sigma=2$ at a very high height but also makes detours to keep zeros of function $\zeta$ on its left even if they are not on the critical line. In order to avoid dramatic growth of the integrand, any detour to the right should be balanced by an almost identical detour to the left. We would then have to deal with huge cancellation between integrals. Although this approach is likely to pose tedious technical problems, it could produce unconditional, accurate results.

\section{REFERENCES}

[Cho95] B.K. Choudhury. The Riemann Zeta-function and its derivative. Proceedings: Mathematical and Physical Sciences, 450(1940):447-499, Sep. 1995. MR1356175 (97e:11095)

[Dec10] A. Decker. Die Widerlegung der Mertens-Vermutung. Mathematisches Institut, Mathematisch-Naturewissenschaftliche Fakultät der Rheinischen Friedrich-WillemsUniversität Bonn, Oct. 2010.

[Kac07] J. Kaczorowski. Results on the Möbius function. J. London Math. Soc., 75(2):509-521, 2007. MR.2340242 (2008g:11162)

[KtR06] T. Kotnik and H.J.J. te Riele. The Mertens conjecture revisited. In Springer, Lecture Notes in Computer Science, volume 4076, pages 156-167, 2006. MR2282922 (2007k:11157)

[LLL82] A.K. Lenstra, H.W. Lenstra, and L. Lovasz. Factoring polynomials with rational coefficients. Mathematische Annalen, 261:513-534, 1982. MR682664 (84a:12002)

[Mer97] F. Mertens. Über eine zahlentheoretische Funktion. Sitzungberichte Akad. Wiss. Wien IIa, (106), 1897.

[OtR85] A.M. Odlyzko and H.J.J. te Riele. Disproof of the Mertens conjecture. J. Reine Angew. Math., (357):138-160, 1985. MR783538 (86m:11070)

[Pin87] J. Pintz. An effective disproof of the Mertens conjecture. Astérique, (147-148):325-333, 1987. MR $891440(88 \mathrm{f}: 11091)$

[RYS69] J.B. Rosser, J.M. Yohe, and L. Schoenfeld. Rigorous computation and the zeros of the Riemann zeta-function. Information Processing 68, Vol 1: Mathematics, Software, pp. 70-76, North-Holland, Amsterdam, 1969. MR0258245(41:2892)

[SD10] Y. Saouter and P. Demichel. A sharp region where $\pi(x)-\operatorname{li}(x)$ is positive. Math. Comp., 79(272):2395-2405, 2010. MR2684372(2011k:11124)

Institut Telecom Brest, BRetagne

E-mail address: Yannick.Saouter@enst-bretagne.fr

CWI, Amsterdam, Netherlands

E-mail address: Herman.te.Riele@cwi.nl 